\title{
A highly interpretable fuzzy rule base using ordinal structure for obstacle avoidance of mobile robot
}

\begin{abstract}
Conventional fuzzy logic controller is applicable when there are only two fuzzy inputs with usually one output. Complexity increases when there are more than one inputs and outputs making the system unrealizable. The ordinal structure model of fuzzy reasoning has an advantage of managing high-dimensional problem with multiple input and output variables ensuring the interpretability of the rule set. This is achieved by giving an associated weight to each rule in the defuzzification process. In this work, a methodology to design an ordinal fuzzy logic controller with application for obstacle avoidance of Khepera mobile robot is presented. The implementation will show that ordinal structure fuzzy is easier to design with highly interpretable rules compared to conventional fuzzy controller. In order to achieve high accuracy, a specially tailored Genetic Algorithm (GA) approach for reinforcement learning has been proposed to optimize the ordinal structure fuzzy controller. Simulation results demonstrated improved obstacle avoidance performance in comparison with conventional fuzzy controllers. Comparison of direct and incremental GA for optimization of the controller is also presented.
\end{abstract}

Keyword: Ordinal structure; Fuzzy logic; Mobile robot; Genetic algorithm; Obstacle avoidance 